Case report

\title{
Porocarcinoma: a rare cause of lateral cervical tumor
}

\author{
Gabriela Maria Aniței ${ }^{*, 1,2}$, Nicolae Șerban loanid ${ }^{2}$, Geanina Bandol ${ }^{3}$, Elena Rodica \\ Gafton $^{4}$, Ana Maria Patrașcu ${ }^{5}$, Dan Ferariu ${ }^{5}$, Angelica Slătineanu ${ }^{6}$, Gema Bugean ${ }^{7}$, \\ Viorel Scripcariu ${ }^{1,2}$
}

1"Grigore T. Popa" University of Medicine and Pharmacy, lasi, Romania; ${ }^{2} 1^{\text {st }}$ Surgical Oncology Unit, Regional Institute of Oncology, lasi, Romania; ${ }^{3}$ Otolaryngology Department, $2^{\text {nd }}$ Surgical Oncology Clinic, Regional Institute of Oncology, lasi, Romania; ${ }^{4}$ Oncology Clinic, Regional Institute of Oncology, lasi, Romania; ${ }^{5}$ Pathology Department, Regional Institute of Oncology, lasi, Romania; ${ }^{6}$ Radiology Department, Regional Institute of Oncology, lasi, Romania; ${ }^{7}$ Radiotherapy Department, Regional Institute of Oncology, lasi, Romania

\begin{abstract}
Porocarcinoma is a rare tumor of the eccrine sweat glands that usually disseminates to the regional lymph nodes, but it can also develop distant metastasis. Case presentation: We report the case of a 67 year-old female patient who underwent wide surgical resection of a left cervical cutaneous tumor in a primary care center, for which the histology exam of the specimen was mixed basal cell and squamous cell carcinoma. She was referred to our hospital's oncology clinic and histologic re-evaluation changed the diagnosis to eccrine porocarcinoma (EPC). Computer-tomography (CT) revealed cervical lymphadenopathies for which the patient underwent 4 cycles of chemotherapy, without regression. She subsequently underwent a left upper anterior jugular lymphadenectomy (group Ila) with all nodes being negative and, three months later, she developed a unique adenopathy under the parotid gland that was excised and confirmed to be metastatic. Postoperative external radiotherapy was administered with a good outcome on CT scan. Nine months after her last surgery, the patient did not show any sign of recurrence or distant metastasis. Conclusion: EPC is a challenge, both diagnostically and therapeutically. In the absence of consensus regarding the indications and extent of lymphadenectomy and adjuvant therapy, patients with EPC should be referred to an experienced multidisciplinary team in a tertiary center.
\end{abstract}

Keywords: eccrine porocarcinoma; skin cancer; sweat glands

\section{Introduction}

Tumors of the skin adnexa are rare, predominantly benign. Almost all of these benign tumor types have a malignant counterpart [1]. Malignant skin adnexal tumors are aggressive, metastasizing in regional lymph nodes or distant organs, or may have

Received: April 2019; Accepted after review: June 2019; Published: June 2019.

${ }^{*}$ Corresponding author: Gabriela Maria Anitei, "Grigore T. Popa" University of Medicine and Pharmacy, 16 Universitatii Str. 700115, Iasi, Romania.

Email: dr.mgabriela@gmail.com recurrence risk $[2,3]$, thus a clear histological diagnosis is extremely important.

Eccrine porocarcinoma (EPC) is a malignant tumor of the eccrine sweat glands, amounting for only $0.005 \%$ of skin cancers [4].

Clinical and histological diagnosis of this type is difficult, as is its yet unstandardized management.

\section{Case report}

A 67 year-old woman was referred to our hospital's oncology clinic for the management of a skin-tumor in the left cervical area who 
underwent surgical resection twelve months before, at a primary care facility. The histology exam she provided described a mixed basal cell and squamous cell carcinoma.

Histological re-evaluation of the paraffin bloc performed in our department of pathology revealed a tumor growth with focal ulcers on its surface, with a solid architecture, cell nests or thick trabecula, some emerging from superjacent epidermis and infiltrating the profound reticular dermis. The tumor was constituted of pleomorphic cells, with roundoval nuclei, some with prominent nucleolus, dispersed chromatin, amphophilic or eosinophilic cytoplasm, with evident cell limits. Small cystic structures were observed focally. Mitotic activity was high - 4-5 mitoses/high power field - and the tumor stroma was fibrous and in low quantity. A moderate

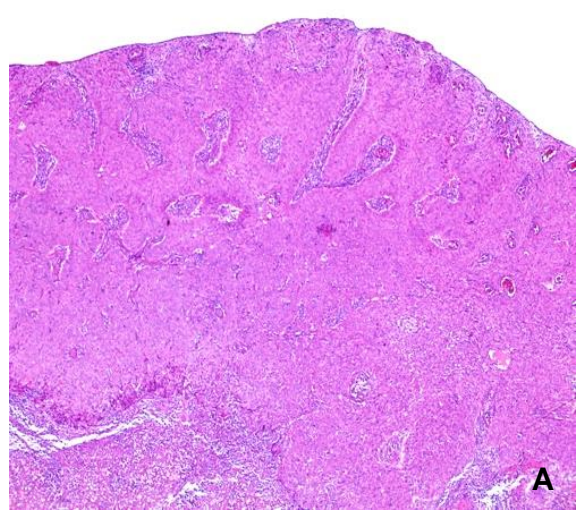

Fig. 1. Eccrine porocarcinoma, histopathology. A: the tumor displays solid and trabecular architecture, with a large growth front (HE, X5); B: major cellular pleomorphism, high mytotic activity, microcysts (HE, X40)

Table 1. Summary of immunohistochemical tests:

\begin{tabular}{|c|c|}
\hline Marker & Description \\
\hline p63 & Diffusely positive in tumor cells \\
\hline EMA & Zonally positive in tumor \\
\hline CK7 & Zonally positive in tumor \\
\hline CEA & Positive in rare tumor cells \\
\hline Bcl-2 & Positive in some tumor cells \\
\hline ER & Weakly positive in rare tumor cells \\
\hline GCDFP15 & Negative \\
\hline
\end{tabular}

EMA=epithelial membrane antigen, CK7=cytokeratin 7, CEA=Carcinoembryonic antigen, Bcl-2=B-cell lymphoma-2 marker, ER=estrogen receptor; GCDFP15= Gross cystic disease fluid protein 15

The patient was re-evaluated with thoracoabdomino-pelvic CT-scan, which excluded secondary lesions. On the other hand, the cervical CT-scan revealed multiple left lateral lympho-plasmocytic inflammatory reaction was observed around the tumor. The profound dermal and hypodermal layer contained satellite tumor nodules with focal necrosis, away from the tumor invasion front (Figure 1). Immunohistochemistry staining of the tumor cells revealed diffuse positivity for p63, zonal positivity for epithelial membrane antigen (EMA) and the low molecular-weight cytokeratin CK7. Carcinoembryonic antigen (CEA) was present in rare tumor cells and the B-cell lymphoma-2 marker (Bcl-2) was positive in some of the tumor cells. Estrogen-receptor was weakly positive in rare neoplastic cells, while the tumor was negative for Gross cystic disease fluid protein 15 (GCDFP15). Immunehisto-chemical analysis is summarized in Table 1. The histology diagnostic was an eccrine porocarcinoma.

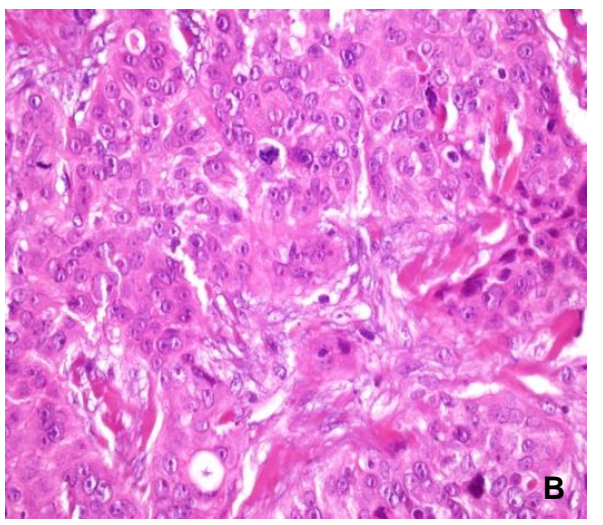


chemotherapy with platinum derivatives and taxanes were administered, after reviewing the literature $[3,5,6]$. However, cervical CT-scan performed two months later showed an important increase in contrast enhancement of one of the left lateral cervical lymphadenopathies, with stationary imaging aspects of the other previously described lymphadenopathies (Figure 2). Given this increased size of cervical lymph nodes, the patient was considered a non-responder to chemotherapy, and surgery was indicated. The patient underwent a wide resection of the previous operative-scar with left latero-cervical lymphadenectomy of the superior compartment (Ila). The cutaneous surgical specimen was tumor free and all of the eleven harvested lymph nodes were negative for metastasis on the histology exam.

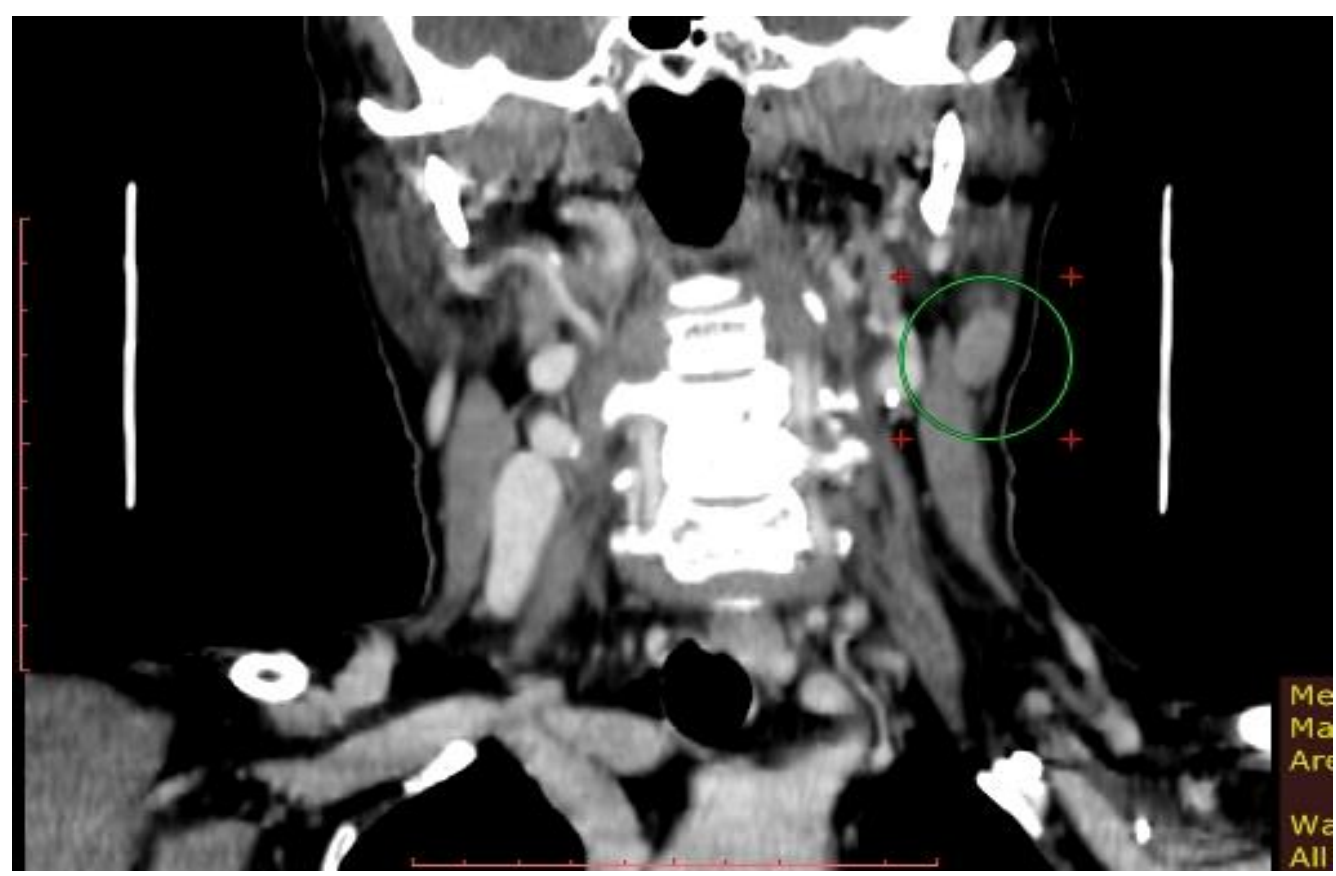

Fig. 2. Cervical CT- scan (coronal view). Left cervical adenopathy (in green circle)

Three months later, as part of the patient's follow-up, a cervical soft-tissue echography revealed a left supero-lateral cervical hypoechogenic lesion, 9/12/9 $\mathrm{mm}$ in size, with Doppler signal present inside, well-defined in contact with the inferior pole of the parotid gland. Two other satellite nodules with similar characteristics were observed, one at the superior pole of the lesion and another at the inferior pole of the lesion, measuring $4 \mathrm{~mm}$ and $3.8 \mathrm{~mm}$ respectively. No lateral cervical lymphadenopathies were observed on either side. The right parotid gland was normal as well.

A positron emission tomography (PET) scan with fluoro-deoxy-glucose (FDG) performed 6 months after surgery described an intense metabolic activity of a left lateral cervical nodular lesion, 14/15/27 mm in size, in contact with the inferior pole of the left parotid gland and the anterior aspect of the left sternocleido-mastoid muscle. No pathological metabolic activity was observed on the lateral cervical nodes bilaterally and the rest of the body. The patient underwent surgical excision of the adenopathy under the parotid gland. Histological examination confirmed the malignant nature of the adenopathy, describing a nodule with extensive metastasis from a carcinoma with solid-type insular architecture, with necrosis in certain comedotype tumor islets. Mitotic activity was evaluated at 7-8 mitosis / high-power field (Figure 3) .

The patient underwent postoperative external radiotherapy $50 \mathrm{~Gy} / 25$ fractions for CTV-N-LR (low-risk nodal clinical target volume) - ipsilateral neck levels Ib, II, III, IV, V, VIII, 60 Gy/30 fractions for CTV-N-HR (high- 
risk nodal clinical target volume) - ipsilateral neck levels II, VIII. Cervical CT-scan after radiotherapy revealed no signs of tumor lymphadenopathies or distant metastasis

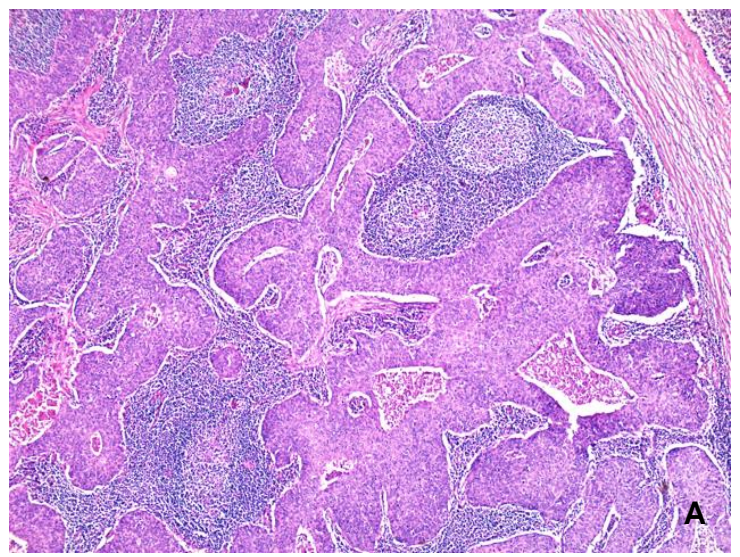

which, according to the data from literature [7, 8], this would indicate a good long-term prognosis.

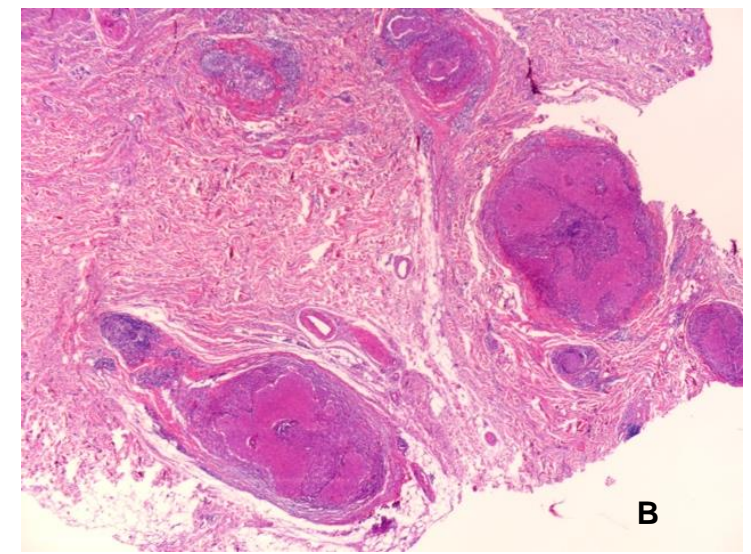

Fig. 3. Metastatic lymphadenopathy. A: lymph node metastasis (HE, x5). B: satellite tumor lymph node (HE, x $2,5)$

\section{Discussions}

Accurate histologic diagnosis of malignant cutaneous adnexal tumors is crucial. However, the rarity of these tumors makes the diagnosis a considerable challenge. The rate of misdiagnosis is around $37-50 \%$ cases analyzed by pathologists in primary care clinics $[9,10]$. Tumor margins can be infiltrative or pushing, the former type being more prone to metastasis and local recurrence. In our case, the tumor margins were infiltrative after first operation that is why the patient underwent a larger resection of the scar.

Robson et al. [11] identify the following features of poor prognosis: more than 14 mitoses/high power field, lympho-vascular invasion and tumor depth $>7 \mathrm{~mm}$. In this case, the mitotic activity was 4-5 mitoses / high power field, without lympho-vascular invasion, this means a good prognosis. However, the profound dermal and hypodermal layer presented satellite tumoral nodules away from the tumor invasion front, which may increase the risk of developing distant metastasis.

Wide-surgical excision is curative in 70$80 \%$ of patients, while the mortality rate in case of metastatic lymphadenopathy is $67 \%$ [12].
Regional lymph node metastases are predominant among secondary lesions (57\%) [13]. Porocarcinoma shows rates of lymphnode metastasis of around 20 percent [14]. However, whether loco-regional lymphadenectomy should always be performed at the time of the primary tumor excision is a matter of debate, some claiming it does not improve the outcome $[6,13]$.

When the diagnosis is certain, the sentinel lymph node biopsy may be performed, but there is not sufficient data to recommend its routine use [5, 14-16]. Regardless of location, the tendency is to perform lymphadenectomy only if there are clinical signs of regional lymph node involvement [17-20].

Barnes et al. [21] found in 69-case series that no malignant adnexal tumor metastasized to the regional lymph nodes before first developing local recurrence.

In our case, the decision for lymphadenectomy was taken given the high probability of metastasis in regional lymph nodes on the CT-scan performed 12 months after resection. Our patient developed a metastasis three months later, in an adjacent lymph node area but still has no cutaneous recurrence by the time of publishing this article.

Stam et al. [14] further recommend, according to melanoma guidelines, adjuvant 
radiotherapy if two or more lymph nodes are metastatic or in case of extracapsular rupture.

Cervical localization of EPC is rare, around $5 \%$ of all EPCs [22], those with lymph node involvement and lymph node dissection even less so. Lymph node cervical groups and subgroups are numerous, thus the extent of cervical lymph node dissection for EPC is prone to considerable variation. Also, EPCs could have a hazardous lymph node metastasis behavior, for example Gutermuth et al. [5] reported only left axillary node involvement for a similar left cervical EPC. Further studies should investigate upon the extent of lymphadenectomy that is required.

The response of skin adnexal tumors to chemotherapy and/or radiotherapy may vary considerably from patient to patient. Patients can survive disease-free for many years after recurrence $[23,24]$ or may conversely have a fulminant oncologic evolution from an apparently early-stage tumor, despite aggressive adjuvant treatment [25]. Our patient had a disease-free interval of 15 months after primary surgery of the skin lesion. By the time of publishing this article, the patient is disease-free 9 months after surgical excision of the lymph node metastasis.

Radiation and chemotherapy (e.g. cyclophosphamide, bleomycin, cisplatin, 5-FU) are not particularly effective and are generally employed for metastatic disease which has an overall survival of 5-24 months [26]. Barzi et

\section{References}

1. Alsaad KO, Obaidat NA, Ghazarian D. Skin adnexal neoplasms-part 1: an approach to tumours of the pilosebaceous unit. $J$ Clin Pathol 2007; 60(2):129-144.

2. Obaidat NA, Alsaad KO, Ghazarian D. Skin adnexal neoplasms-part 2: an approach to tumours of cutaneous sweat glands. J Clin Pathol 2007; 60(2):145-159.

3. Oyasiji T, Tan W, Kane J, et al. Malignant adnexal tumors of the skin: a single institution experience. World J Surg Oncol 2018; 16(1):99.

4. Riera-Leal L, Guevara-Gutiérrez E, BarrientosGarcía JG, Madrigal-Kasem R, Briseño- al. [27] obtained good results with isotretinoin and interpheron-alpha.

Because of the low mitotic activity and negative thoraco-abdomino-pelvic CT-scan for distant metastasis after 9 months from the surgical excision of the nodule situated in the inferior pole of the parotid gland, we may say that the patient has a good prognosis.

\section{Conclusion}

Eccrine porocarcinoma is a rare and aggressive malignant skin adnexal tumor with particular diagnostic challenges. There is no consensus regarding the need for systematic regional lymphadenectomy and its extent. Further evidence is needed also to address the issue of adjuvant therapy strategy in case of metastatic and/or recurrent eccrine porocarcinoma. The authors' opinion is that EPC would be best treated in a tertiary center by an experienced multidisciplinary team.

\section{Consent}

Written informed consent was obtained from the patient for publication of this case report and accompanying images. A copy of the written consent is available for review by the Editor-in-Chief of this journal.

\section{Competing interests}

The authors declare that they have no competing interests.

Rodríguez G, Tlacuilo-Parra A. Eccrine porocarcinoma: epidemiologic and histopathologic characteristics. Int J Dermatol 2015; 54(5):580-586.

5. Gutermuth J, Audring $\mathrm{H}$, Voit C, Trefzer U, Haas N. Antitumour activity of paclitaxel and interferon-alpha in a case of metastatic eccrine porocarcinoma. J Eur Acad Dermatology Venereol 2004; 18(4):477-479.

6. De luliis F, Amoroso L, Taglieri L, et al. Chemotherapy of rare skin adnexal tumors: a review of literature. Anticancer Res 2014; 34(10):5263-5268.

7. Kurashige $Y$, Minemura $T$, Nagatani $T$. Eccrine 
porocarcinoma: clinical and pathological report of eight cases. Case Rep Dermatol 2013; 5:259-266

8. Prieto-Granada C, Castner N, Chen A, et al. Behavior of cutaneous adnexal malignancies: a single institution experience. Pathol Oncol Res. 2018 [doi: 10.1007/s12253-018-0427-3].

9. Luz M de A, Ogata DC, Montenegro MFG, Biasi LJ, Ribeiro LC. Eccrine porocarcinoma (malignant eccrine poroma): a series of eight challenging cases. Clinics (Sao Paulo) 2010; 65(7):739-742.

10. Belin E, Ezzedine K, Stanislas S, et al. Factors in the surgical management of primary eccrine porocarcinoma: prognostic histological factors can guide the surgical procedure. $\mathrm{Br} J$ Dermatol 2011; 165(5):985-989.

11. Robson A, Greene J, Ansari N, et al. Eccrine porocarcinoma (malignant eccrine poroma): a clinicopathologic study of 69 cases. Am J Surg Pathol 2001; 25(6):710-720.

12. Marone U, Caracò $C$, Anniciello $A M$, et al. Metastatic eccrine porocarcinoma: report of a case and review of the literature. World J Surg Oncol 2011; 9:32.

13. Salih AM, Kakamad $\mathrm{FH}$, Baba $\mathrm{HO}$, et al. Porocarcinoma; presentation and management, a meta-analysis of 453 cases. Ann Med Surg 2017; 20:74-79.

14. Stam $H$, van de Wiel BA, Klop WMC, et al. Skin adnexal carcinoma of the head and neck: a retrospective study in a tertiary referral center. Eur Arch Oto-Rhino-Laryngology 2015; 272(4):1001-1010.

15. Shiohara J, Koga H, Uhara H, Takata M, Saida T. Eccrine porocarcinoma: Clinical and pathological studies of 12 cases. J Dermatol 2007; 34(8):516-522.

16. Sahn RE, Lang PG. Sentinel lymph node biopsy for high-risk nonmelanoma skin cancers. Dermatologic Surg 2007; 33(7):786793.

17. Chang $O$, Elnawawi A, Rimpel B, Asarian A, Chaudhry N. Eccrine porocarcinoma of the lower extremity: A case report and review of literature. World J Surg Oncol 2011; 9:94.

18. Ramasenderan $\mathrm{N}$, Shahir $\mathrm{H}$, Omar SZ. A synchronous incidence of eccrine porocarcinoma of the forearm and facial squamous cell carcinoma: A case report. Int $J$ Surg Case Rep 2018; 42:116-120.

19. Walsh MS. A case of eccrine porocarcinoma. $J$ $R$ Soc Med 1990; 83(8):529-530.

20. Orella Jal, Penalba Av, Juan Ccs, Nadal Rv, Morrondo Jc, Alvarez Tt. Eccrine porocarcinoma. Dermatologic Surg 1997; 23(10):925-928.

21. Barnes M, Hestley A, Murray DR, Carlson GW, Parker D, Delman KA. The risk of lymph node involvement in malignant cutaneous adnexal tumors. Am Surg 2014; 80(3):270-274.

22. Urso $C$, Bondi $R$, Paglierani $M$, Salvadori $A$, Anichini C, Giannini A. Carcinomas of sweat glands: report of 60 cases. Arch Pathol Lab Med 2001; 125(4):498-505.

23. González-López MA, Vázquez-López F, Soler $\mathrm{T}$, et al. Metastatic eccrine porocarcinoma: a 5.6-year follow-up study of a patient treated with a combined therapeutic protocol. Dermatol Surg 2003; 29(12):1227-1232.

24. Ponzetti A, Ribero S, Caliendo V, Spadi R, Macripò G, Lista P. Long-term survival after multidisciplinary management of a metastatic sarcomatoid porocarcinoma with repeated exeresis, radiotherapy, chemotherapy and cetuximab: case report and review of literature. G Ital Dermatol Venereol 2017; 152(1):66-70.

25. Fujimine-Sato $A$, Toyoshima $M$, Shigeta $S$, et al. Eccrine porocarcinoma of the vulva: a case report and review of the literature. J Med Case Rep 2016; 10(1):319.

26. Sawaya JL, Khachemoune A. Poroma: a review of eccrine, apocrine, and malignant forms. Int J Dermatol 2014; 53(9):1053-1061.

27. Barzi AS, Ruggeri S, Recchia F, Bertoldi I. Malignant metastatic eccrine poroma. Proposal for a new therapeutic protocol. Dermatol Surg 1997; 23(4):267-272. 